\title{
La pratique de l'injection solide
}

Y. IAGOLNITZER

A. MONNET

R. KATZENBACH

Bachy, TH Darmstadt (Allemagne)
L'injection solide, technique d'amélioration de sol utilisée depuis une quinzaine d'années en France, est basée sur l'incorporation dans le sol sous haute pression d'un mortier mi-sec. S'engraissant en masse sans imprégner ni claquer le terrain, le mortier refoule le sol environnant et en améliore ainsi les caractéristiques géotechniques. Réalisées à partir de forages de petit diamètre par passes en remontant, des inclusions de mortier sont formées, dont la répartition en maillage apporte une amélioration supplémentaire.

L'étendue des possibilités d'application est très vaste, puisque l'injection solide est envisageable dans tous les sols compressibles des sables aux argiles. Les types d'application comprennent aussi bien les reprises en sous-cuvre et réfections de fondations de toutes natures, que l'amélioration de portance, le traitement de terrains karstiques, ou la réduction du potentiel de liquéfaction.

\section{Compaction grouting practice}

Compaction grouting, a soil improvement method that has been used for the past 10 years 10 years in France, is based on the incorporation of a low water content mortar in the ground under high pressure. Growing as a mass without impregnating or fracturing the soil, the mortar displaces the surrounding soil and thus improves its geotechnical properties. From small diameter holes and staging upwards, mortar columns are formed, their distribution in a regular pattern bringing an additionnal improvement.

The broadness of the application field is very large, as compaction grouting may be envisaged in all compressive soils ranging from sands to clays. The applications include underpinning and stabilisation of all types of foundations, as well as bearing capacity improvement, treatment of karst terrains, or lowering of the liquifaction index. 


\section{Introduction}

On ne présente plus aux États-Unis l'injection solide, devenue presque traditionnelle à la suite de près de cinquante ans d'expériences positives et généralisées. Née et développée dans le sud-ouest des ÉtatsUnis à la fin des années 40, cette technique d'amélioration et de renforcement des sols lâches, basée sur l'incorporation de mortier sous haute pression, comptait déjà en 1975 plusieurs centaines de chantiers réussis (Bowen, 1975). En 1989, lors du tremblement de terre de Loma Prieta qui ébranla San Francisco, aucun désordre n'est reporté sur les 200 à 300 structures ayant auparavant fait l'objet d'un traitement par injection solide (Graf, 1992).

A l'origine, l'injection solide permettait de stabiliser et remettre à niveau des fondations d'ouvrages ayant subi des tassements différentiels. Par rapport à l'injection classique, elle assurait un meilleur contrôle de la localisation du matériau et par conséquent une meilleure maîtrise du traitement et de ses effets. L'éventail des applications s'est par la suite largement étendu, en grande partie grâce à l'adaptabilité de la technique, tant aux conditions de sol qu'aux éventuelles structures existantes.

Introduite dans notre pays par la société Bachy en $1983, l^{\prime}$ injection solide a donc douze ans d'expériences en France, où elle fait encore partie des «techniques nouvelles m.

Malgré les succès qu'elle remporte dans ses applications, la généralisation de la technique en France et en Europe est relativement lente. Les deux raisons principales en sont les suivantes:

- La première concerne les pays francophones. Il est clair que l'existence de cinq appellations différentes pour une technique nouvelle est un frein à son développement. En effet, alors que la dénomination américaine compaction grouting est unique, simple et sans équivoque, ce que nous appelons « injection soldie m ou " compactage par injection solide » est appelé par Solétanche " compactage statique horizontal », et en amalgame proposé par le CEBTP, «le compactage statique horizontal par injection solide ») abrégé CSHIS (ainsi que «compactage statique par injection solide horizontale) ! ; enfin, on trouve le terme américain utilisé entre guillemets, ainsi que sa traduction littérale : injection de compactage. Les références bibliographiques sont difficiles à rassembler, l'idée que l'on se fait de la technique est nécessairement floue. Il est temps aujourd'hui de faire un choix. Puisque Bachy, dans ses priorités de recherche, a réservé à l'injection solide ces dernières années une place importante et parce que le terme " injection solide » est à la fois simple et imagé, même s'il ne contient pas la notion de "compactage"), c'est ce dernier que nous proposons.

- La deuxième raison concerne l'ensemble de l'Europe. Tous les aspects de la technique sont aujourd'hui encore empiriques, aucune forme de réglementation ou de recommandation, ni américaine, ni française ni européenne n'existe à ce jour. Or, lorsqu'une technique (marche», mais qu'elle n'est pas étayée scientifiquement, même si les mécanismes de fonctionnement semblent avoir été percés, elle n'est pas toujours assez convaincante. L'expérience remplace ce manque de support scientifique, encore faut-il avoir accès à cette expérience. En France, les maitres d'œuvre et d'ouvrage sont parfois ouverts aux techniques nouvelles, et il faut ici saluer leur courage et leur esprit d'innovation. C'est ce qui a permis le développement de l'injection solide en France, outre, bien sûr, les éléments nouveaux et intéressants qu'elle apporte, qu'ils soient d'ordre économique, technique, environnemental ou écologique. Dans d'autres pays, où une technique doit, pour être mise en œuvre, posséder un cadre normatif régissant dimensionnement, exécution et contrôles, comme c'est le cas en Allemagne par exemple, l'injection solide ne pouvait voir le jour,

alors que certaines entreprises allemandes ont déjâ utilisé la technique à l'étranger !

Un vaste programme de recherche, soutenu par la Commission des communautés européennes a été lancé sur ce sujet, dans le cadre d'une collaboration entre I'entreprise Bachy et I'Institut de géotechnique de l'Université de Darmstadt (Allemagne). L'objet de cet article n'est pas de traiter tout le problème, mais d'en poser les bases en présentant un état de l'art de la pratique de l'injection solide. En essayant de dévoller les hypothèses des mécanismes implicites qui la sous-tendent, il vise donc à décrire la technique telle qu'elle est pratiquée aujourd'hui et s'appuie, lorsque c'est nécessaire sur son histoire. Après avoir présenté le principe de l'injection solide, l'équipement et la mise en œuvre seront exposés. On abordera les sols et les types d'application, illustrés d'exemples de réalisations américaines et françaises.

\section{2}

\section{Principe et mécanismes}

L'injection solide a tout d'abord été utilisée dans la remise à niveau de structures. C'est en comparant les volumes de mortier incorporés à ceux strictement nécessaires aux relèvements obtenus que l'on a pris conscience qu'un certain volume de mortier avait refoulé et donc compacté le sol environnant. Le principe de l'injection solide était né.

A un niveau de traitement élémentaire, l'injection solide consiste donc à incorporer dans le sol un mortier suffisamment sec et frottant pour former une masse homogène qui s'engraisse tout au long de l'incorporation. Il n'y a pas de mélange entre le matériau et le sol, mais une interface nette. Le mortier ne remplit pas les pores du terrain, ne le claque pas, mais le refoule. Le sol est densifié par compaction.

Cette philosophie est née dès les premières applications de remises à niveau de structures, qui prohibaient tout soulèvement brutal, donc tout claquage. Mais de manière générale, l'absence de claquage est justifiée par la recherche d'une maitrise de l'injection: I'ouverture de claquages entraîne la perte du contrôle de l'injection. La localisation du mortier n'est plus certaine, des résurgences et des soulèvements peuvent se produire. Ces derniers nécessitent l'arrêt immédiat de l'injection, aucun compactage supplémentaire ne pouvant plus être obtenu. C'est la qualité et l'efficacité du traitement qui sont alors remises en cause, car l'arrêt de l'injection se produit en général avant qu'il ait été possible d'injecter les quantités nécessaires au compactage désiré.

D'autres philosophies, qui atteignent globalement le même objectif de compaction, se sont développées 
plus tard en se basant au contraire sur les claquages: c'est le cas de l'injection de fracturation (fracturing grouting).

L'absence de claquages étant un des principes fondamentaux de la technique, il convient ici de préciser ce que Yon entend exactement sous cette appellation: un claquage est une rupture du sol due à un déséquilibre des forces créé par l'injection sous pression. Cette rupture se produit au droit du forage, selon un plan: elle est alors définie par sa longueur et sa largeur maximale. Elle peut dans certains cas se produire apres infiltration du mortier dans des fissures ou discontinuités du terrain déjà existantes, qui constituent des chemins privilégiés. La forme et l'orientation de l'ouverture vont déterminer si le mortier jouera le rôle de vérin plat et soulèvera le terrain, ce qui arrive lorsque l'ouverture est horizontale et large. Les formes de claquages qui ne risquent pas d'occasionner de soulèvements en surface sont bénins, comme c'est le cas à grande profondeur. Les claquages sont le plus souvent verticaux, comme on le constate couramment en injection classique, mais aussi en injection solide ; cela vient du fait qu'une rupture se produit dans un plan perpendiculaire aux contraintes principales minimales, le plus souvent horizontales. Il n'en reste pas moins qu'on ne maitrise pas, à l'échelle d'une passe, l'orientation d'un claquage, dont on cherche par conséquent à se prémunir.

La formation, à partir d'une boule déjà formée, d'une excroissance s'engraissant à sa périphérie, n'est pas considérée comme un claquage. Il en est de mème pour les galettes qui peuvent se former à l'interface de deux couches de terrains.

Les causes de claquages sont nombreuses et interdépendantes:

- le terrain: la présence de fissures ou de points de faiblesses dans le sol, un drainage insuffisant dans les terrains argileux saturés :

- la profondeur du traitement: près de la surface ou à proximité d'une pente, où la pression de confinement peut devenir insuffisante ;

- le mortier: une trop grande fluidité du mortier, ses propriétés rhéologiques

- les paramètres d'injection: une vitesse ou une pression instantanée d'injection trop élevée.

Mais une telle sujétion serait vaine sans movens de contrôles, qui peuvent être exercés

- en déterrant les inclusions; c'est une mesure après coup qu'il est bien difficile de relier à l'injection ellemême. Elle donne cependant une bonne idée générale :

- pendant l'incorporation, en observant le paramètre de la pression d'injection: une diminution brutale de la pression indique en général un claquage. Les résurgences sont le signe d'un mortier très liquide, souvent susceptible de claquer le terrain. La consistance du mortier est par ailleurs contrôlée régulièrement par l'essai d'affaissement au cône d'Abrams.

Le mécanisme de compactage induit par l'injection solide, d'acceptation unanime aujourd'hui aux ÉtatsUnis, est le suivant : l'incorporation provoque l'apparition d'un système complexe de contraintes radiales et tangentielles. La figure 1 montre une zone de remaniement importante au contact de la masse de mortier, qui se traduit par des cisaillements et des déformations plastiques. Plus loin, le sol est dans un état de déformations élastiques. L'étendue de la zone d'influence dépend:
- des caractéristiques du terrain (nature, teneur en eau, compacité);

- du matériau injecté (caractéristiques rhéologiques); - des paramètres d'incorporation (débit, pression, quantités injectés).

C'est thypothèse de la zone de remaniement qui permet d'expliquer les observations peu compréhensibles faites au moyen de mesures de densité, où le sol éloigné apparaissait plus densifié que le sol directement au contact de l'inclusion.

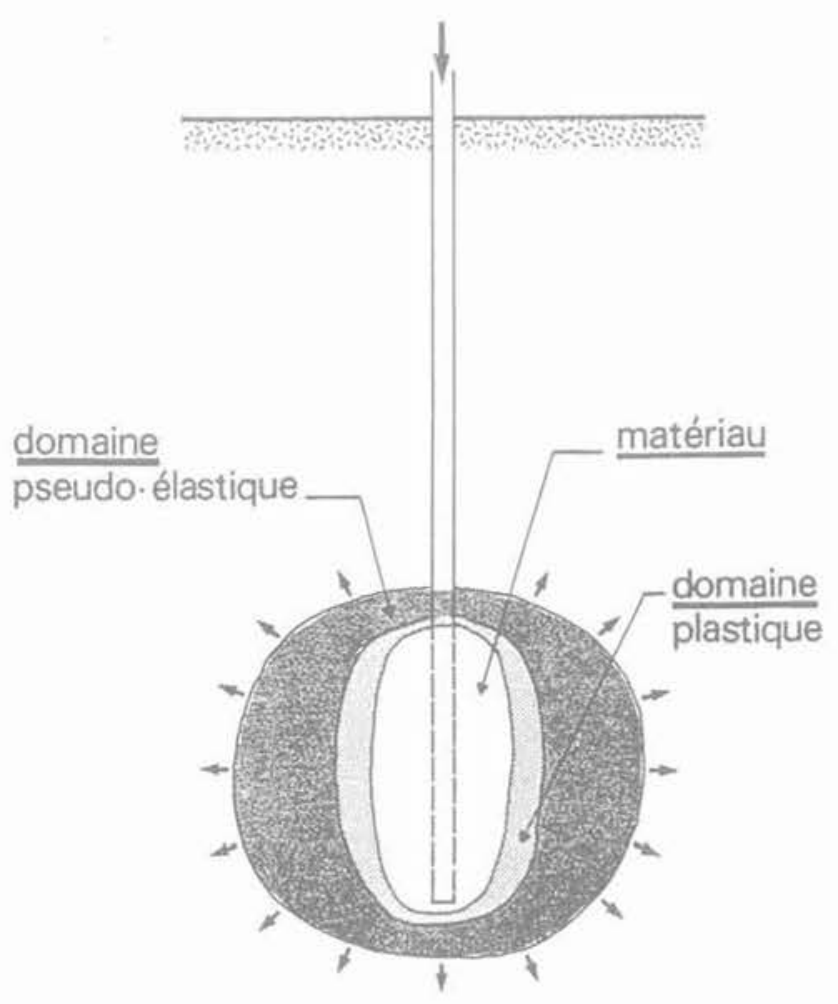

HG. 1

Effet de compactage du sol par engraissement d'une boule de mortier. Compactive effect through a growing mortar body.

En fait, ces phénomènes s'expliquent simplement par les principes de base de la mécanique des sols: si l'on considère un petit volume de sol à la périphérie d'un forage et sa déformation après incorporation de mortier, il parait clair que ce volume de sol, extrêmement étiré, est dans un état plastique. Les déformations diminuant avec la distance du point d'injection, il arrive une distance à partir de laquelle les déformations ne sont plus plastiques, mais élastiques. Le sol à la périphérie de la boule de mortier est en rupture jusqu'à une certaine distance du point d'injection, où il est donc le siège de cisaillements importants.

Une explication de la formation d'une zone de moindre densité à la périphérie de la boule de mortier peut être touvée dans le phénomène connu de la dilatance, du moins dans les sols granulaires: tous y sont en effet sujet, si la contrainte de confinement est suffisamment faible, or l'injection solide s'applique justement aux sols lấches. Mais il reste à montrer que cette hypothèse est bien vérifiée dans tous les cas. 
Quant aux sols cohérents, l'augmentation de la pression interstitielle entraîne une déconsolidation du terrain à proximité de la boule de mortier, qui peut être à l'origine de la chute de densité.

A un niveau de traitement général, les boules de mortier sont réalisées les unes au-dessus des autres pour former dans le sol des inclusions, verticales ou inclinées.

La quantité de mortier globale à incorporer est déterminée en fonction de l'amélioration de sol recherchée, plus précisément en fonction d'une densité de sol moyenne à atteindre. Elle est en général exprimée sous la forme d'un taux d'incorporation, variable entre 2 et $12 \%$ du volume du terrain traité, avec une moyenne de $5-6 \%$.

La quantité de mortier est ensuite répartie régulièrement sur la surface à traiter, selon un maillage. Ce dernier est défini en fonction de l'étendue du domaine d'influence que l'on peut atteindre dans le terrain concerné, avec les conditions d'injection prévues, et compte tenu des quantités prédéterminées. Cette phase est encore arbitraire et empirique.

La réalisation des inclusions de mortier avec les quantités prédéterminées et suivant le maillage défini mène à :

- une amélioration des caractéristiques intrinsèques du terrain par compactage ;

- et un renforcement par la présence d’un groupe d'inclusions de mortier.

L'injection solide allie donc les deux types de traitements que sont l'amélioration et le renforcement des sols. Selon le type d'application, la nature du sol et ses caractéristiques mécaniques, l'un des effets peut être prédominant par rapport à l'autre.

\section{3}

\section{Mise en œuvre}

\section{1}

\section{Équipement}

La figure 2 montre l'équipement classique d'un chantier d'injection solide:

-1 unité de préparation du mortier (malaxeur, centrale de chantier ou livraison par camion toupie);

-1 malaxeur de reprise :
- 1 pompe pouvant injecter à un débit réglable entre 15 et $150 \mathrm{l} / \mathrm{mm}$ (ou 1 à $10 \mathrm{~m}^{3} / \mathrm{h}$ ) à $8 \mathrm{Mpa}$ de pression maximum. Graf (1992) évoque même des pompes allant jusqu'à 7-11 Mpa;

- 1 foreuse.

C'est un équipement réduit et léger, qui rend la technique très adaptable et lui permet par exemple de travailler sous faible hauteur ou à proximité immédiate d'existants.

Si les relevés des paramètres de forage et d'injection se faisaient encore manuellement lors des débuts de l'injection solide, ils sont aujourd'hui entièrement automatisés sur tous les chantiers, et participent au contrôle en temps réel des travaux. Les paramètres les plus couramment enregistrés sont:

- En phase de forage: la vitesse d'avance, la pression d'injection du fluide de perforation et la pression sur l'outil en fonction de la profondeur;

- pendant l'incorporation: le débit, la pression et les quantités de mortier en fonction du temps, ceci à chaque profondeur de passe.

Il est à noter que l'enregistrement de la pression, pris en France à la pompe, l'est également au point d'injection aux États-Unis.

La pompe est la pièce maîtresse de l'équipement. C'est d'ailleurs au développement de pompes à haute pression que l'injection solide doit le sien, comme Bowen (1975) en fait le passionnant historique. En effet, ces pompes permettaient d'envisager de pousser un matériau extrêmement frottant, qui se comportait très différemment dans le sol des coulis d'injection classique.

Ses caractéristiques principales, exposées par Warner (1992) sont les suivantes : c'est une pompe à piston possédant une isolation par valve étanche entre le cylindre et le gaveur. Il n'existe pas sur le marché de pompe spécialement conçue pour l'injection solide. On utilise en général une pompe à béton qu'il est nécessaire de modifier. En effet, les pompes à béton classiques ont des valves qui fuient, ce qui ne porte pas à conséquence pour le béton car la course du piston est rapide et les pressions modérées. En revanche, l'utilisation de telles pompes, insuffisamment adaptées, en injection solide, où la course est lente et les pressions très élevées, peut provoquer des problèmes: en général le blocage du mortier après perte d'eau.

Francescon et Twine (1992) conseillent que les pompes ne produisent pas de surcharges de pression ou pics, comme peuvent le faire les pompes de type piston réciproque. Ils pensent aussi que le volume de chaque coup de piston et la vitesse de pompage, en liai-

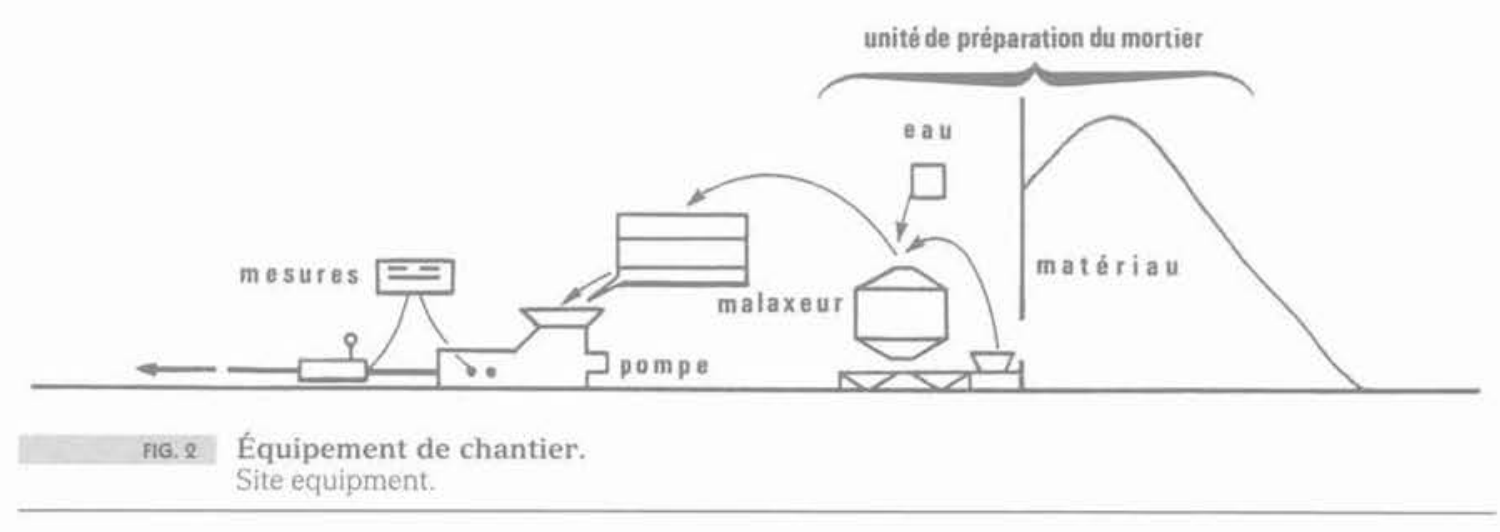


son avec le volume de chaque passe d'injection, peuvent affecter les risques de claquage du terrain.

Si la pompe constitue l'élément central, il faut noter que tous les raccords, joints..., situés sur le parcours du mortier entre le malaxeur de reprise et le forage sont des emplacements naturels de fuites, qu'il convient de limiter en nombre, et de bien étancher afin d'éviter les problèmes de blocage de mortier.

\section{2}

\section{Mise en œuvre élémentaire}

Elle concerne un forage individuel, et au sein de ce forage une passe d'injection.
La mise en œuvre de I'injection solide se fait en deux phases, forage et injection suivant le schéma de la figure 3 .

Aux États-Unis, l'injection solide est pratiquée le plus souvent en descendant, selon les étapes suivantes : - forage en diamètre supérieur jusqu'au haut de la zone à traiter ou 1,2 $\mathrm{m}$ de la surface;

- scellement au coulis de ciment du tubage métallique; - forage à travers le tubage pour extension du forage sur la hauteur d'une passe :

- injection d'une passe:

- généralement le jour suivant, reforage de la passe précédente et extension du forage;

- injection de la passe suivante, etc.

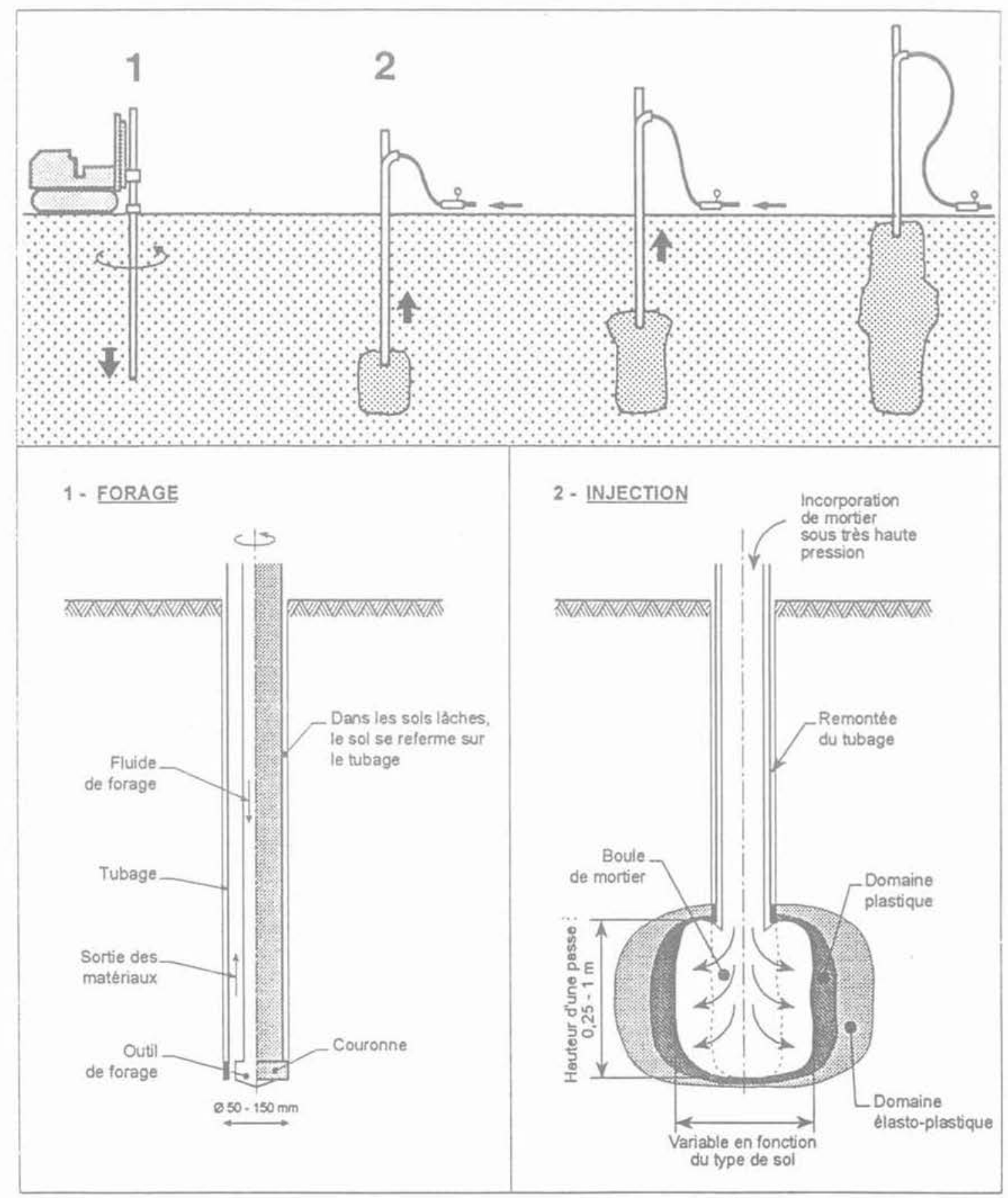

FIG. 3 Mise en œuvre de l'injection solide. Execution of compaction grouting. 
La technique a été reprise en Europe en remontant, pour des raisons évidentes de rapidité et d'économie, les passes étant injectées les unes après les autres sans délai.

Les principales différences sont:

- le besoin d'une couverture de terrain (horizontalement et verticalement) plus importante dans l'injection en remontant. Lorsqu'on ne bénéficie pas de la présence de surcharge ou d'une couche de surface de bonne qualité, $3 \mathrm{~m}$ sont nécessaires au lieu de $1,2 \mathrm{~m}$. Cette sujétion représente la limitation majeure de la technique;

- les inclusions créées n'ont pas la même forme; ce sont de véritables colonnes par la méthode en descendant, alors que l'injection en remontant forme plutôt un chapelet de bulbes. Leur fonctionnement est a priori différent.

Cependant, les deux méthodes sont aussi efficaces, comme Graf (1992) en a fait l'observation sur des centaines de chantiers.

Stilley (1982) note, quant à lui, que la méthode en descendant semble plus adaptée pour la stabilisation et la remise à niveau de fondations.

\section{4}

\section{Préparation}

\section{- Forage}

En France, le forage est réalisé avec tubage simultané, en petit diamètre (100 mm environ), le plus souvent en rotation, mais cela dépend des ourrages ou des terrains de surface à traverser.

Le forage est descendu jusqu'à la profondeur maximale du traitement.

\section{- Prétraitement}

Ce sujet a été évoqué par Brown et Warner (1973), Warner et al. (1974), Warner (1982). Dans les sols très fins, le fluide de perforation a parfois été traité avec un agent mouillant, afin de réduire la résistance au cisaillement du sol autour du point d'injection, d'éviter l'essorage du mortier et son blocage pendant l'incorporation et donc de faciliter l'injection et d'augmenter les quantités injectables, On obtient le même effet en remplissant le forage d'eau avant injection: l'augmentation des pressions interstitielles lors de l'injection fait en effet chuter la contrainte effective et abaisse le seuil de cisaillement (Saley et al., 1987); cette dernière pratique possède l'inconvénient évident d'augmenter la teneur en eau du sol.

Dans les sols argileux, des coulis à base de chaux hydratée ou d'autres agents chimiques ont été utilisés pour améliorer la résistance de la masse du sol. Notons qu'il n'est plus fait mention de prétraitement dans la littêrature américaine de la masse du sol. Est-ce parce qu'elle s'est banalisée ou qu'elle a disparu? Il reste que son efficacité n'avait été évaluée que vis-à-vis des quantités de mortier incorporables, mais ni par rapport à l'amélioration de sol, ni au regard de la qualité, et en particulier de l'augmentation potentielle des risques de claquages. L'utilisation d'un prétraitement nécessite donc des recherches complémentaires,

\section{- Armature}

En fin de forage, on peut introduire une barre métallique (en général de diamètre $2,5 \mathrm{~cm}$ ), ce qui est pratiqué systématiquement pour certains types d'application (Graf, 1992).

\section{0 \\ Injection}

L'injection est concentrée dans la zone intéressée, le forage étant simplement rempli de mortier au droit des terrains ne nécessitant pas de traitement.

\section{- Débit d'incorporation}

Warner (1982) distingue le débit initial, compris entre 20 et $30 \mathrm{l} / \mathrm{mn}$, d'un débit courant ( optimal » défini expérimentalement sur site, comme étant le débit le plus rapide permettant de maintenir l'augmentation de la pression dans une fourchette de 35 à $50 \mathrm{kPa}$ par minute. Ce débit sera variable suivant les problèmes et les terrains à traiter, maís dépassera rarement $80 \mathrm{l} / \mathrm{mm}$. Warner (1982) donne encore un exemple de débit de $15 \mathrm{l} / \mathrm{mn}$ dans le cas d'un sol très peu perméable.

Pour Graf (1992), le débit doit être réduit à $20 \mathrm{l} / \mathrm{mn}$ lorsqu'on arrive près de la pression de refus.

Les récents essais semblent confirmer le rôle important du débit d'incorporation pour la qualité des travaux et en particulier vis-à-vis de l'ouverture des claquages, mais on manque encore de résultats pour fixer des limites précises.

\section{- Pertes de charge}

Conformément au principe de la technique, une grande partie de la pression initiale est dissipée en pertes de charge. On cherche autant que possible à les minimiser, en réduisant la distance et donc la longueur des conduites entre la pompe et les forages (100 m est un maximum) et en évitant les raccords coudés. Les pertes de charge augmentent avec la vitesse de pompage, mais pas linéairement: d'après une observation de Francescon et Twine (1992), elles doublent pour une vitesse de pompage multipliée par 4.

\section{- Passes}

Elles sont comprises entre 0,25 et $1 \mathrm{~m}$ en France, 1,5 et $2,5 \mathrm{~m}$ aux États-Unis.

Graf (1992) évoque une pratique américaine qu'il juge conservatrice consistant à prendre pour hauteur de passe la demi-maille.

La hauteur de passe influence la forme des bulbes de mortier, qui seront plutôt sphériques pour des hauteurs de passes réduites, plutôt cylindriques pour des hauteurs de passes plus importantes.

\section{- Critères d'arrêt}

L'injection est immédiatement arrêtée lorsque l'un des critères suivants est atteint :

- déplacement maximal admissible du sol ou d'un ouvrage, vertical ou horizontal (dont l'évaluation nécessite instrumentation et contrôles adéquats). Parfois, ce phénomène est prévisible car il se produit une chute de pression brutale (de 0,35 à $0,5 \mathrm{MPa}$ ), indiquant un claquage du sol. Graf (1992) en fait même un critère d'arrêt préliminaire.

A moins de constituer le but du traitement, les soulèvements sont nocifs et impliquent l'arrêt immédiat de 
l'injection, car le terrain ne peut plus être compacté, mais risque en revanche d'être destructuré (Warner, 1982):

- pression maximale de refus (hors pertes de charge); - quantité prédéterminée (de 2 à $12 \%$ suivant le terrain et le cas à traiter).

Ce critère n'est pas le même pour tous les forages: il dépend du phasage (voir § 3.3 .2 ). Souvent, les quantités sont limitées en primaire, plus rarement en secondaire. Cela permet de répartir les quantités de mortier de manière homogène.

Graf (1992) évoque une pratique utilisêe dans le cas de projets critiques: attente de 3 minutes après la première injection, puis réinjection (de la même passe). On répète cette procédure jusqu'à ce que le sol prenne moins de $50 \%$ que la quantité à la phase précédente.

Quelques projets ont été menés avec des critères d'arrêt variables en fonction de la profondeur comme une augmentation de la pression et une diminution des quantités injectées.

\section{3}

\section{Mise en œuvre globale}

Elle concerne les paramètres généraux nécessaires à l'exécution: quantités à incorporer, maillage, phasage d'injection, pression et débit, ainsi que la composition du mortier. Il est en général nécessaire de procéder à un plot d'essai au début ou avant le chantier, afin d'affiner tous ces paramètres.

La figure 4 donne la représentation d'une incorporation de mortier courante pour une maille de $9 \mathrm{~m}^{2}$.

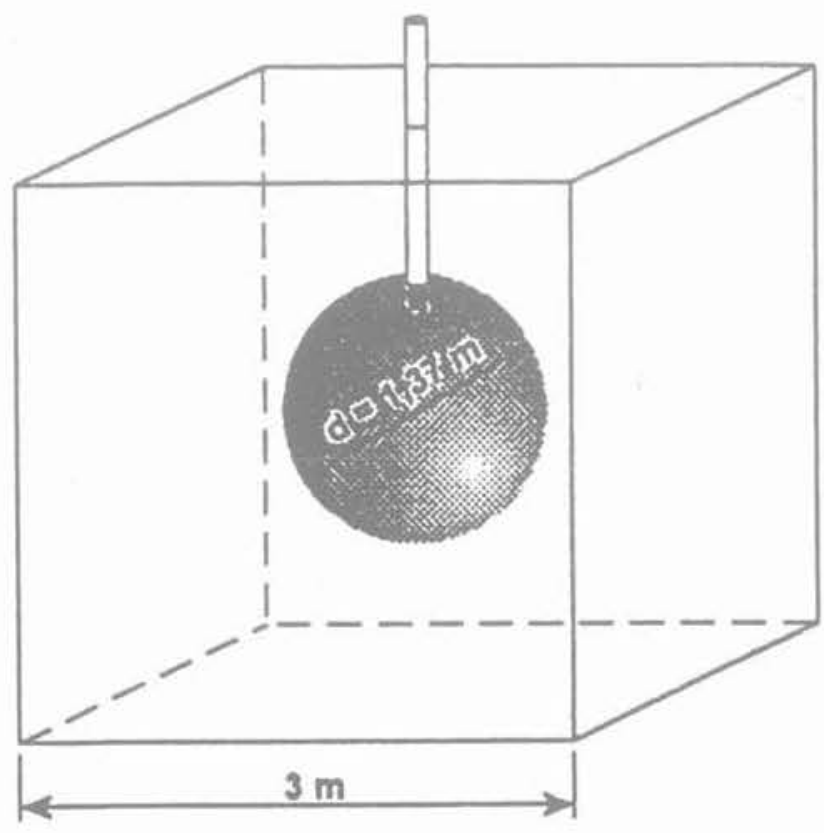

FG.4 Représentation d'une incorporation de mortier de $5 \%$ avec une maille de $9 \mathrm{~m}^{2}$. Representation of a $5 \%$ mortar incorporation with $9 \mathrm{~m}^{2}$ grid.

\section{7}

\section{Maillage}

Le but de l'injection solide étant de compacter le sol en tout point, et aucune méthode de calcul simple n'ayant encore été développé pour déterminer l'effet de compactage en tout point, la maille est aujourd hui fixe par l'évaluation empirique de l'étendue du domaine d'influence de l'injection solide dans un sol donné, compte tenu des quantités globales estimées. Le domaine d'influence n'a pas de géométrie précise, puisque la boule de mortier s'engraisse préférentiellement en refoulant les zones de faibles résistances. De manière simplifiée, on utilise pour sa projection en plan le terme de rayon d'action. Généralement, le rayon d'action, et donc la maille, dépendent:

- du sol : nature, teneur en eau, densité relative ;

- des paramètres d'incorporation : débit, pression et quantités :

- de la profondeur du traitement;

- de l'environnement et des objectifs à atteindre.

Warner (1982) précise que dans la plupart des cas, la densification atteint une zone entre 0,3 et $1,8 \mathrm{~m}$ à partir de l'interface bulbe de mortier/sol.

La maille est couramment comprise entre 1,5 et $5 \mathrm{~m}$. Compte tenu de la taille des inclusions (de 0,3 à $0,9 \mathrm{~m}$ de diamètre), ces valeurs sont cohérentes avec les rayons d'actions cités par Warner.

Voici quelques indications pratiques. D'une manière générale, la maille est serrée dans les terrains proches de la surface, ainsi que dans le cas de travaux à proximité d'existants ou de reprise en sous-ceuvre.

L'expérience montre que la maille peut être plus lâche dans les sols lâches que dans les sols plus compacts car les bulbes en terrains lâches sont beaucoup plus gros.

De manière générale, on observe un rayon d'action moindre dans les terrains sableux que dans les terrains argileux.

On trouve dans la littérature l'opinion que, pour un même traitement, la maille peut être élargie en profondeur. Dans la pratique, seuls de rares chantiers ont fait l'objet d'une telle mesure, ce qui s'explique par la complexité d'exécution, et par le fait que la possibilité d'appliquer une plus forte pression est en partie compensée par l'augmentation de la résistance passive du terrain.

Les formes de maille les plus variées sont utilisées : carrées, triangulaires, en diamant, en nid d'abeille... Les conditions d'exécution étant un aspect capital sur les chantiers, et l'influence de la forme de maille sur la qualité du traitement étant minime, on conseillera plutôt une maille carrée. On peut définir la maille finale moyenne comme le quotient de la surface traitée augmentée de la demi-maille ayant cours par le nombre total de forages. Dans le cas de forages inclinés, c'est la surface moyenne définie comme ci-dessus au milieu de la profondeur de traitement, qui est à prendre en compte.

\section{3atas}

\section{Phasage}

L'injection se fait traditionnellement en primairesecondaire, parfois tertiaire. Cette attitude aujourd'hui systématique joue un rôle essentiel dans le contrôle en 
cours de travaux de l'efficacité et de la qualité du traitement: on a vu que la maille représentait d'une certaine façon le rayon d'action anticipé de l'injection solide. Par conséquent, si maille et quantités prédéterminées sont bien dimensionnées, on doit observer en fin d'injection des forages secondaires, entourés de forages déjà traités, une forte augmentation des pressions associée à un ralentissement de la vitesse d'injection.

Lors de la confortation d'ouvrage, il est préférable de commencer par les points de traitement intérieurs si l'on veut éviter les soulèvements et/ou chasser des matériaux très lâches encombrant des vides (Vezinhet et al., 1990).

Dans les autres cas, la réalisation des forages périphériques en premier (toujours en primaire-secondaire), donne de meilleurs résultats, car la zone à traiter est encagée et bénéficie d'un effet d'étreinte latérale. Cela s'applique d'autant plus que le traitement s'effectue sur une faible largeur. Cette mesure est aussi nécessaire lorsque le traitement est réalisé à proximité d'ouvrages à protéger. Des précautions particulières (resserrement de la maille, quantités et débits diminués) sont prises pour l'injection des forages périphériques, qui forment une barrière pour la suite des travaux.

\section{4}

\section{Domaines d'application}

\section{1}

\section{Sols}

Au début, l'injection solide permettant de traiter des terrains considérés comme ininjectables, on limita l'application de l'injection solide aux sols plus fins qu'un sable moyen.

En réalité, tous les sols granulaires peuvent être améliorés grâce à l'injection solide, comme on ne tarda pas à s'en apercevoir. Comme le dit Graf très logiquement, tous les sols pouvant être compactés par évacuation d'eau ou d'air aux vitesses utilisées peuvent être traités par injection solide.

Ainsi, toute la gamme de sols de l'argile au sable, de même que les sols organiques comme la tourbe ont déjà fait l'objet de traitements par injection solide.

Des remblais d'argile hors nappe ont déjà pu être densifiés. Par contre, l'efficacité du traitement est encore contreversée dans les argiles saturées: Graf (1992) met en garde contre les surpressions d'eau qui peuvent nécessiter plusieurs jours à se dissiper, Guilloux et al. (1989) conseillent la mise en place d'un réseau de drainage, tandis que Warner (1982) prône la diminution de la vitesse d'injection.

En ce qui concerne les matériaux décomposables (décharges), il est clair qu'à moins d'un traitement chimique associé permettant de les neutraliser, l'effet de compactage obtenu ne peut être que provisoire.

En France, on décrit plus volontiers le type de sol par sa pression limite $\mathrm{p}_{1}$ au pressiomètre ou sa résistance de pointe $q$ au pénétromètre statique que par sa granulométrie: Dupeuble et al. (1985) observent que tous les sols lâches dont la pression limite est inférieure à $0,5 \mathrm{MPa}$ ou, dont la résistance de pointe est inférieure à 2-4 Mpa, peuvent être traités par injection solide.
Gambin (1985) et Robert (1989) vont plus loin en donnant des limites en fonction du type de sol:

$-\mathrm{p}_{1}<0,3 \mathrm{MPa}, \mathrm{Em}<3 \mathrm{Mpa}, \mathrm{q}_{\mathrm{c}}<1 \mathrm{MPa}$ dans les terrains argileux;

$-\mathrm{P}_{1}<0,6 \mathrm{MPa}, \mathrm{Em}<6 \mathrm{MPa}, \mathrm{q}_{c}<4 \mathrm{MPa}$ dans les terrains limoneux;

$\mathrm{p}_{1}<1,2 \mathrm{MPa}, E \mathrm{Em}<12 \mathrm{Mpa}, \mathrm{q}_{\mathrm{c}}<12 \mathrm{MPa}$ dans les terrains sableux.

Dans les terrains hétérogènes, cette technique offre un avantage particulier en ce sens que les zones les plus lâches reçoivent plus de mortier et sont donc plus compactés (Guilloux et al., 1989). Outre une amélioration générale, on bénéficie également d'une homogénéisation des caractéristiques du terrain.

Il convient enfin de mentionner tous les terrains de type karstique. Ce sont des sols dont la matrice est dure, mais qui présentent des vides (de dissolution, ou crées par l'homme dans le cas des mines) plus ou moins comblés par des fragments ou débris de la matrice, de la vase ou encore de l'eau. Ce sont les terrains où l'injection solide s'adapte particulièrement bien, car elle permet dans le même temps de combler les vides sans déperdition de mortier et de recompacter les zones décomprimées.

\section{2}

\section{Types d'application}

Les domaines d'application sont présentés par ordre de fréquence d'articles, et on peut supposer de fréquence d'occurence de chantiers.

- Reprise en sous-œuvre ou confortation de fondations d'ouvrages ayant subi des tassements. Le traitement peut être envisagé avec ou sans remise à niveau.

C'est à l'origine pour ce type d'application qu'est née l'injection solide, qui permettait beaucoup plus de précision que l'injection classique dans la localisation du matériau injecté, et donc dans le contrôle de la densification du sol.

Les remises à niveau peuvent atteindre $50 \mathrm{~cm}$, avec une précision de $3 \mathrm{~mm}$ (parfois même $1,5 \mathrm{~mm}$ ). Il convient de préciser que, dans le cas de hauteurs de soulèvement importantes, on utilise des vérins hydrauliques, le vide créé étant comblé en fin de traitement.

Graf (1992) rapporte le cas de remise à niveau de la charge la plus concentrée, pour une autoroute surélevée : 500 t sur une semelle carrée de $4,5 \mathrm{~m}$ de côté (hors surcharges).

On peut citer deux cas particuliers intéressants que sont l'amélioration de la butée de pied d'une paroi et l'amélioration de la résistance de pointe ou de frottement latéral de pieux, où trois forages d'injection solide sont réalisés autour des pieux (Warner, 1982).

Les canalisations ou les caniveaux enterrés peuvent faire l'objet d'un traitement soit pour minimiser des déformations futures soit pour prévenir des désordres en cas d'augmentation de charge (Warner, 1982) et (Graf, 1992).

Les tirants n'ayant pas donné de bons résultats aux essais de traction peuvent aussi être renforcés par injection solide (Graf, 1992).

- Amélioration de la portance d'un sol dans le cas de construction neuves, ou d'augmentation de 
charge sur des fondations existantes, afin de limiter les tassements absolus et différentiels (immeubles courants, réservoirs, remblais, culées d'ouvrages...).

- Traitement de terrains karstiques ou de mines abandonnées: l'injection solide permet de combler les vides, de recompacter les zones décomprimées, et de bloquer les fontis. C'est une application intéressante pour les réseaux ferroviaires, routiers ou autoroutiers.

- Réduction du potentiel de liquéfaction d'un sol, dans le but d'une protection sismique.

- Injection de compensation, correspondant au compensation grouting anglais, qui désigne un traitement, actuellement en injection classique ou solide, destiné à corriger au fur et à mesure les affaissements de terrain en surface dûs à l'avancement d'un tunnelier dans un sol meuble. Cette technique est en général utilisée en site urbain. L'injection s'arrête entre 1,8 et 2,5m au-dessus de la voûte.

Lorsque l'injection solide ne peut ètre pratiquée depuis la surface, on peut forer et injecter soit à travers le revêtement du tunnel à l'arrière du tunnelier, soit à partir de puits latéraux.

- Stabilisation de glissements (Guilloux et Blondeau, 1989). Surtout adaptée aux glissements dont la surface se trouve en profondeur.

- Fondation d'ouvrages : Graf (1992) évoque l'utilisation de colonnes d'injection solide en tant que piliers et colonnes portantes: des exemples de structures de soutènement ont déjà été rapportés.

Les encadrés suivants présentent brièvement cinq réalisations correspondant aux types d'applications les plus répandues. Ces exemples ont été choisis pour leur originalité, afin de montrer la diversité des situations et des conditions d'utilisation de la technique. Ils sont issus du Génie civil, mais les exemples d'applications sont assez bien répartis avec le Bâtiment.

L'injection solide peut être utilisée conjointement à d'autres techniques d'amélioration de sol, comme le compactage dynamique, le drainage dans les sols peu perméables, ou encore l'injection classique, dans les sites karstiques. L'encadré $n^{\circ} 3$ présente un exemple d'encagement en injection solide et de noyau en injection classique.

\section{5}

\section{Conclusion}

Par sa grande adaptabilité, l'éventail des applications possibles et par le fait qu'elle conjugue amélioration et renforcement des sols, l'injection solide est amenée à se développer en Europe. Les succès remportés jusqu'à présent et la fréquence croissante des applications lui faisant appel en sont une bonne indication.

Le principe d'engraissement d'une boule de mortier, I'une des bases fondamentales de la technique, est mis en pratique grâce à un équipement adéquat, mais repose surtout sur l'expérience, la compétence et le sérieux du personnel d'entreprise. Afin de limiter le caractère empirique de la technique, ainsi que l'importance des plots d'essai en amont ou en début de chantier, il est aujourd'hui nécessaire d'approfondir la compréhension des mécanismes en jeu pour développer des méthodes de dimensionnement et de définition des paramètres de traitement.

\section{Bibliographie}

Baker W.H. - Embankment foundation densification by compaction grouting, Issues in Dam Grouting. Proc, of an ASCE Geotech. Eng. Div, session, Denver, Colorado, 1985 , p. 104-122.

Bowen R. - "Compaction Grouting $n$ in Grouting in Engineering Practice, John Wiley \& Sons, N.Y., 1975, p. 65-77.

Brown D.R., Warner J. - Compaction Grounting. Journal of Soll Mechanics and Foundations Division. Proc. of the ASCE, vol. 99, SM8, Proc. Paper 9908. aoút 1973 , p. 589-601.

Byle M.J. - Limited Compaction grouting for Retaining Wall Repairs, Grouting. Soil improvement and Geosynthethics, ASCE Geotech. Special Publi., $n^{\circ} 30$. New Orleans, 1992, p. 288-300.

Critchfield J.W., Macdonald J.F. - Seattle Bus Tunnel Construction, Rapid Excavation and Tunnelling Conference, Los Angeles, 1989, ch. 22, p. 341-359.

Dupeuble P., Robert J., Deniau A. - Le compactage par injection solide, Travaux, juillet-aoút 1985, p. 1-8.

Francescon M. Twine D. - Treatment of solution features in upper chalk by com- paction grouting, Proc, of the ICE Grouting in the Ground Conference, London, ed. Bell, 1992 , paper 20, p. 327-347.

Gambin M. - Tendances nouvelies en amélioration des sols, Chantiers de France n*186, décembre 1985, p. 1-10.

Graf E.D. - Compaction grout, 1992. Février 1992, Grouting, Soll improvement and Geosynthetics, ASCE Geotech. Special Publi., $n^{\circ} 30$, New Orleans, 1992, p. 275-287.

Guilloux A., Blondeau F. - Le traitement des sols fins par injection solide, Proc. of the XIIth internat. Conf. on SMFE, Rio de Janeiro, 1984, vol. II, p. 13671368 .

Robert J. - Amélioration des sols par intrusion de mortier, Proc. of the XIIth Internat. Conf. on SMFE, Rio de Janeiro, 1989, vol. II, p. $1407-1408$.

Robinson R.A., Kucker M.S., Parker H.W. - Ground behavior in glacial soils for the Seattle Transit Tunnels, Rapid Excavation and Tunnelling Conference, 1991. p. 93-117.

Salley J.R., Foreman B., Baker W.H. Henry J. - Compaction Grouting Test
Program - Pinopolis West Dam, Soil Improvement - A ten year update, ASCE Geotechnical Special Publication $n^{\circ} 12,1987$, p. 245-269.

Stilley A.N. - Compaction grouting for foundation stabilization, Conference on Grouting in Geotechnical Engineering, New Orleans, Ed. W.H. Baker ASCE. New York, 1982, p. 923-937.

Vezinhet A., Londez M., Kretz A., Stenne R. Confortement du sol sous une longrine de voie de portique - Terminal minéralier du Port Autonome de Marseille, Travaux, avril 1990, p. 38-44.

Warner J. - Compaction Grouting - The first thirty years, ASCE Symposium New Orleans, 1982, p. 694-707.

Warner J. - Compaction grout-Rheology vs. effectiveness, Grouting, Soil improvement and Geosynthetics, ASCE Geotech. Special Publi., $n^{\circ} 30$, New Orleans, 1992, p. 229-239.

Warner J., Brown D.R. - Planning and Performing Compaction Grouting, Journal of the geotechnical engineering division, Proc. of the ASCE, vol. 100, GT6, Proc. Paper 10606, juin 1974, p. 653-666. 


\section{APPLICATION 1 : REPRISE EN SOUS-0EUVRE}

\section{Chantier}

Bair Ranch Interchange, Interstate 70, Colorado, Etats-Unis.

\section{Maître d'ouvrage}

Colorado Division of Highways.

\section{But de l'intervention}

Arrêter l'évolution des mouvements de tassements ( $35 \mathrm{~cm}$ en 3 ans pour 15 à 20 prévus) et de déversement d'un mur de soutènement.

\section{Ouvrage}

Mur de soutènement en panneaux de béton préfabriqués en double $T$ supportant la voie principale de l'Interstate 70. Mur fondé sur une semelle de répartition et remblayé à l'arrière. Les charges totales varient de 75 à $140 \mathrm{kPa}$.

\section{Terrains}

Un préchargement devait permettre l'accélération du tassement de la couche d'argile lacustre. Les tassements étant plus élevés que prévu, des tests complémentaires ont été réalisés, dont des essais de consolidation après immersion montrant la sensibilité des sables limoneux aux augmentations de teneur en eau sous charge, sur $6 \mathrm{~m}$ de hauteur.

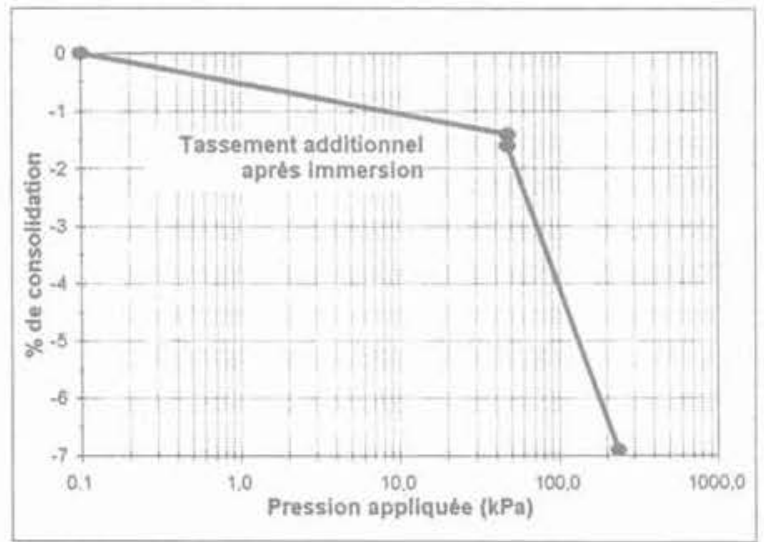

Test de consolidation

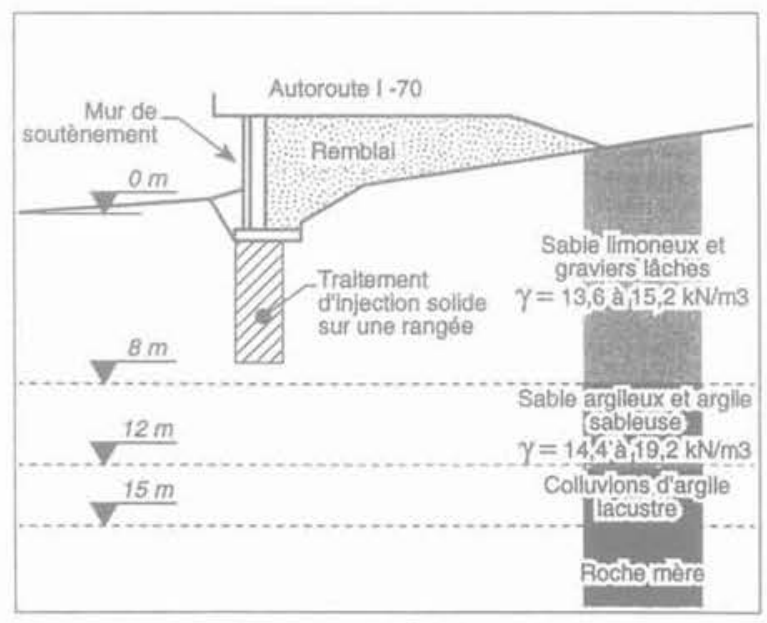

Coupe sur l'ouvrage

\section{Traitement}

Amélioration du système de drainage du mur, et réalisation d'une ligne d'injection solide sous le pied de la paroi de 0 a $6 \mathrm{~m}$ de profondeur. Forages espacés de $1.5 \mathrm{~m}$ (2 points d'injection par panneau en $T$ ).

\section{Quantités}

Forages : $230 \mathrm{U}$

Linéaire foréfinjecté : $1380 \mathrm{ml}\left(230^{*} 6\right)$

Volume de mortier incorporé : $443 \mathrm{~m}^{3}$

Taux d'incorporation (sur une bande de $2,4 \mathrm{~m}$ de largeur)

- dans les 2,4 m supérieurs : $4,5 \%$

- dans les $3,6 \mathrm{~m}$ inférieurs : $10,7 \%$ moyenne : $8.2 \%$

Réduction de l'indice des vides : $11 \%$

\section{Résultats}

pas de tassements significatifs depuis la fin des travaux.

\section{Commentaires}

injection mixte en descendant sur les $2,4 \mathrm{~m}$ supérieurs, puis en remontant. Utilisation de bentonite prohibée.

Données issues de Byle, (1992) 


\section{APPLICATION 2 : AMELIORATION DE PORTANCE D'UN SOL}

\section{Chantier}

Darse 1, postes Q1 et Q2 du Terminal minéralier de Fos-sur-mer, France.

\section{Maître d'ouvrage}

Port Autonome de Marseille.

\section{But de l'intervention}

Augmenter la capacité portante de $290 \mathrm{kN} / \mathrm{ml}$ à 500 $\mathrm{kN} / \mathrm{ml}$ pour le nouveau portique de déchargement, avec une limitation de flèche pour la longrine de $4 \mathrm{~mm}$.

\section{Terrains}

- Matelas de cailloutis sur $2,5 \mathrm{~m}$.

- Sables fins moyennement compacts sur $20 \mathrm{~m}$. très lâches par endroits.

\section{Quantités}

\section{Forages : $814 \mathrm{U}$}

Linéaire foré/injecté : $7074 / 5358 \mathrm{ml}$

Volume de mortier incorporé : $1574 \mathrm{~m}^{3}$

Taux d'incorporation :

moyenne : $5,4 \%$

\section{Résultats}

\begin{tabular}{|c|c|c|c|}
\hline & \multicolumn{3}{|c|}{ Résistance de pointe (MPa) } \\
\hline & Avant & Après & $\begin{array}{c}\text { Facteur d’augmentation } \\
\text { moyen }\end{array}$ \\
\hline 01 & $\begin{array}{l}3 \cdot 6 \\
\text { moy. } 4,1\end{array}$ & $\begin{array}{c}5 \cdot 13 \\
\text { moy } 7,3\end{array}$ & 1,8 \\
\hline $\mathrm{Q} 2$ & $\begin{array}{l}6 \cdot-8,5 \\
\text { moy, } 6,8\end{array}$ & $\begin{array}{l}7-15 \\
\text { moy. } 10,6\end{array}$ & 1,55 \\
\hline
\end{tabular}

Comparaison de résultats d'essais pénétrométriques

Essai de chargement composé de 5 cycles courts de 2 heures puis d'un cycle long de 2 jours : toutes les fiecches mesurées s'avérent inférieures aux valeurs maximales fixées au C.C.T.P.

\section{Commentaires}

- Maintien du quai en exploitation pendant les travaux

- utilisation d'un mortier san's ciment.

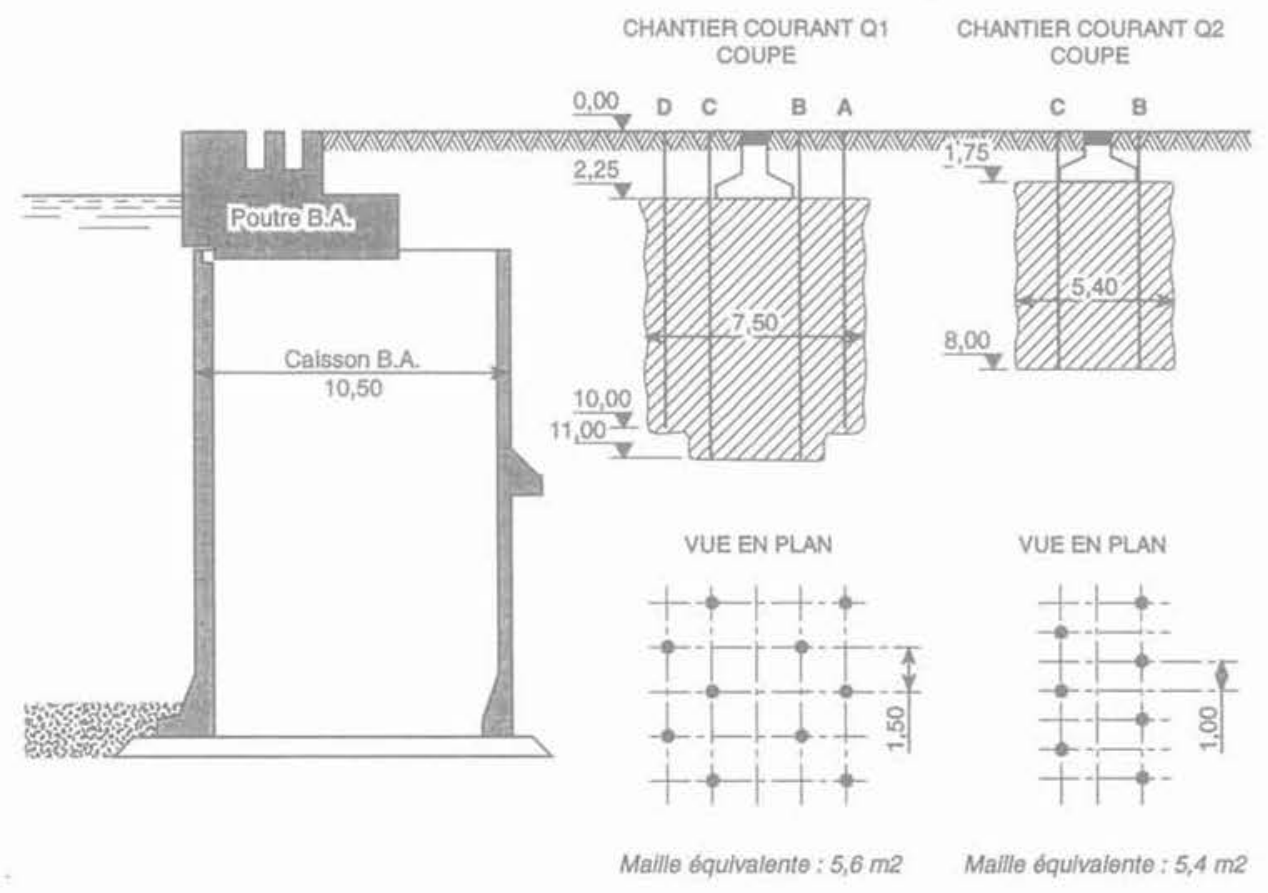

Données issues de Vezinhet et al., (1990)

Application 2 Amélioration de portance d'un sol.

Bearing capacity improvement. 


\section{APPLICATION 3 : TRAITEMENT DE TERRAINS KARSTIQUES}

\section{Chantier}

Pont d'Arcy sur Cure, RN 6, Pile $n^{\circ} 6$, France.

\section{Maître d'ouvrage}

D.D.E. d'Auxerre Sud-Est.

\section{But de l'intervention}

Remplir les cavités et fissures du terrain et de la maçonnerie de fondation et recompacter les zones de remplissage karstiques décomprimées, afin d'assurer la stabilité à long terme de la pile et du pont.

\section{Terrains}

Les calcaires compacts abondamment faillés du Jurassique, siège de fractures élargies par dissolution.

\section{Traitement}

- par injection solide : encagement de la pile sur $20 \mathrm{~m}$ de hauteur et traitement en profondeur (entre 8 et $20 \mathrm{~m}$ ) du noyau.

- par injection classique : traitement du noyau de surface et de la maçonnerie.

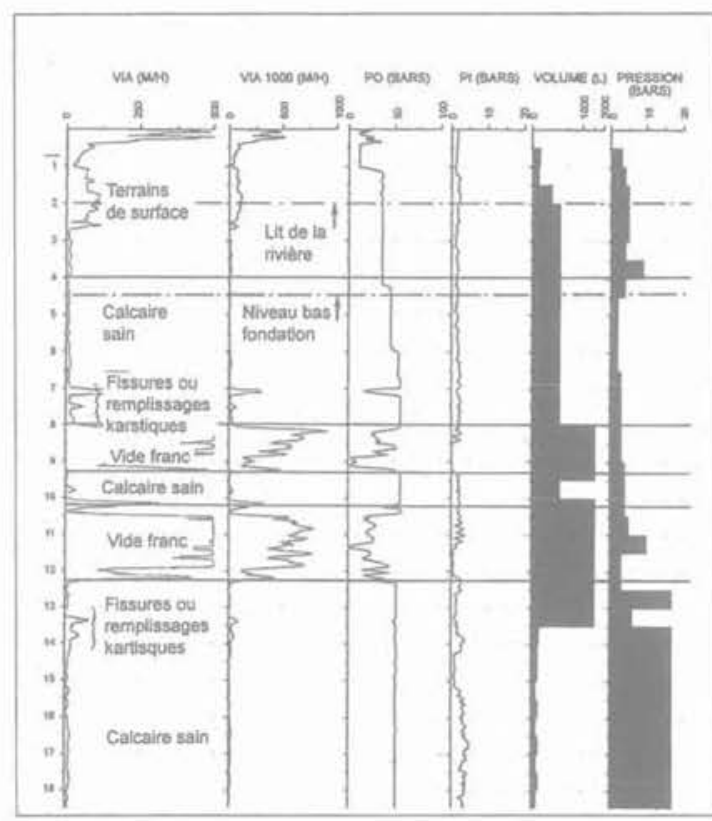

Enregistrement des paramètres de forage et synthèse des paramètres d'incorporation

\section{Commentaires}

- Travail sous hauteur réduite

- Des traitements analogues sont en ce moment en cours sur d'autres piles du pont présentant les mêmes symptômes.

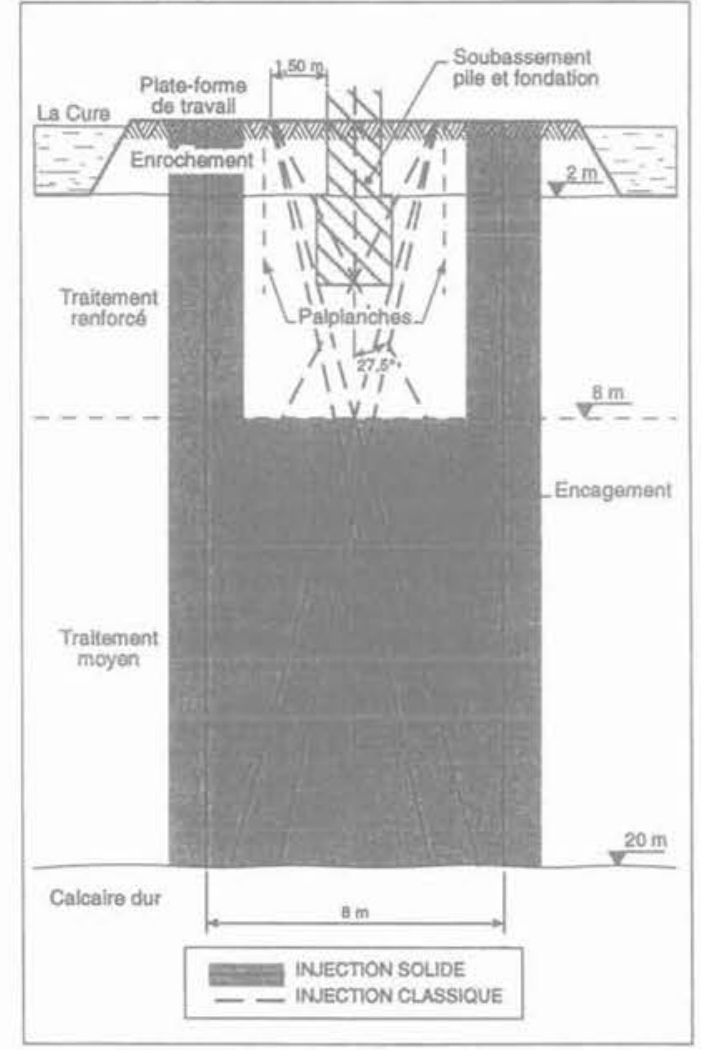

\section{Quantités}

Coupe type

\section{Forages : $51 \mathrm{U}$}

Linéaire foréfinjecté : $1030 \mathrm{ml}$

Volume de mortier incorporé : $433,5 \mathrm{~m}^{3}$

Taux d'incorporation :

- dans les vides francs $110 \%$

- dans les matériaux de remplissage karstique $55 \%$,

- dans le calcaire sain $3 \%$

moyenne : $13 \%$

\section{Résultats}

Des essais pressiométriques mettent en évidence l'amélioration des caractéristiques de sol et des essais Lefranc permettent de contróler la faible perméabilité, qui assure une limitation des circulations d'eau et par conséquent la pérennité du traitement :

\begin{tabular}{|c|c|c|c|c|c|c|}
\hline \multirow{3}{*}{ Protondeur } & \multicolumn{4}{|c|}{ PAgSTON } & \multirow{2}{*}{\multicolumn{2}{|c|}{ 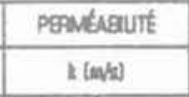 }} \\
\hline & \multicolumn{2}{|c|}{$\alpha(0(0)$} & \multicolumn{2}{|c|}{$5(\mathrm{PP})$} & & \\
\hline & OBJECTF & REGUSt: & DQuectif & RÉLSt & OQUECTF & Réuster \\
\hline $2.4 \mathrm{~m}$ & 0.8 & 1.4 & 4.8 & 13 & -104 & 104 \\
\hline $4.5 \mathrm{~m}$ & 0.5 & 3.5 & 4,0 & 25 & & \\
\hline $6.8 \mathrm{~m}$ & 0.4 & 3.0 & 3,2 & 32 & & \\
\hline $8.10 \mathrm{~m}$ & . & 3,0 & . & 32 & & \\
\hline$>10 \mathrm{~m}$ & 0,3 & 3,0 & 2.4 & 32 & $\begin{array}{l}\text { - Volaur: } \\
\text { « Vheur: }\end{array}$ & $\begin{array}{l}5 \text { mitimum } \\
5 \text { meoimem }\end{array}$ \\
\hline
\end{tabular}




\section{Chantier}

Barrage de Pinopolis West (plot d'essai), Caroline du Sud, Etats-Unis.

\section{Maître d'ouvrage}

Savannah District Corps of Engineers

\section{But de l'intervention}

Densifier une couche de sables låches sous nappe située sous le barrage en terre $(21,3 \mathrm{~m}$ de hauteur et $2011 \mathrm{~m}$ de longueur) pour réduire la sensibilité sismique.

\section{Terrains}

- 0-9 m sables compacts.

- 9-12 m sables lâches.

- Marnes moyennement compactes.

\section{Traitement}

Sur une surface de $620 \mathrm{~m}^{2}$, le traitement est concentré sur la couche de sables láches. La maille finale est de $1,8 \mathrm{~m}$, atteinte en 3 phases.

\section{Quantités \\ Forages : $120 \mathrm{U}$ \\ Linéaire injecté : $170 \mathrm{ml}$ \\ Volume de mortier incorporé : $123 \mathrm{~m}^{3}$ \\ Taux d'incorporation moyen : $12.7 \%$}

\section{Résultats}

\begin{tabular}{|c|c|c|c|}
\hline & Avant & Après & Objectif \\
\hline $\begin{array}{c}\mathrm{SPT} \\
(\mathrm{nb} \text { coups } / \mathrm{SO} \mathrm{cm})\end{array}$ & $0=7$ & 17 & 11 \\
\hline $\begin{array}{c}C P T \\
q \subset \text { (MPa) }\end{array}$ & 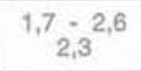 & 7,9 & \\
\hline $\begin{array}{l}\text { DILATOMETRE } \\
\text { Module (MPa) } \\
\text { Indice de contrainte } \\
\text { horizontale Ko }\end{array}$ & $\begin{array}{c}9,7 \\
1\end{array}$ & $\begin{array}{l}66,2 \\
4,5\end{array}$ & \\
\hline Densité sèche & $1,36=1,6$ & & 1,68 \\
\hline
\end{tabular}

Amélioration de la couche de sables láches

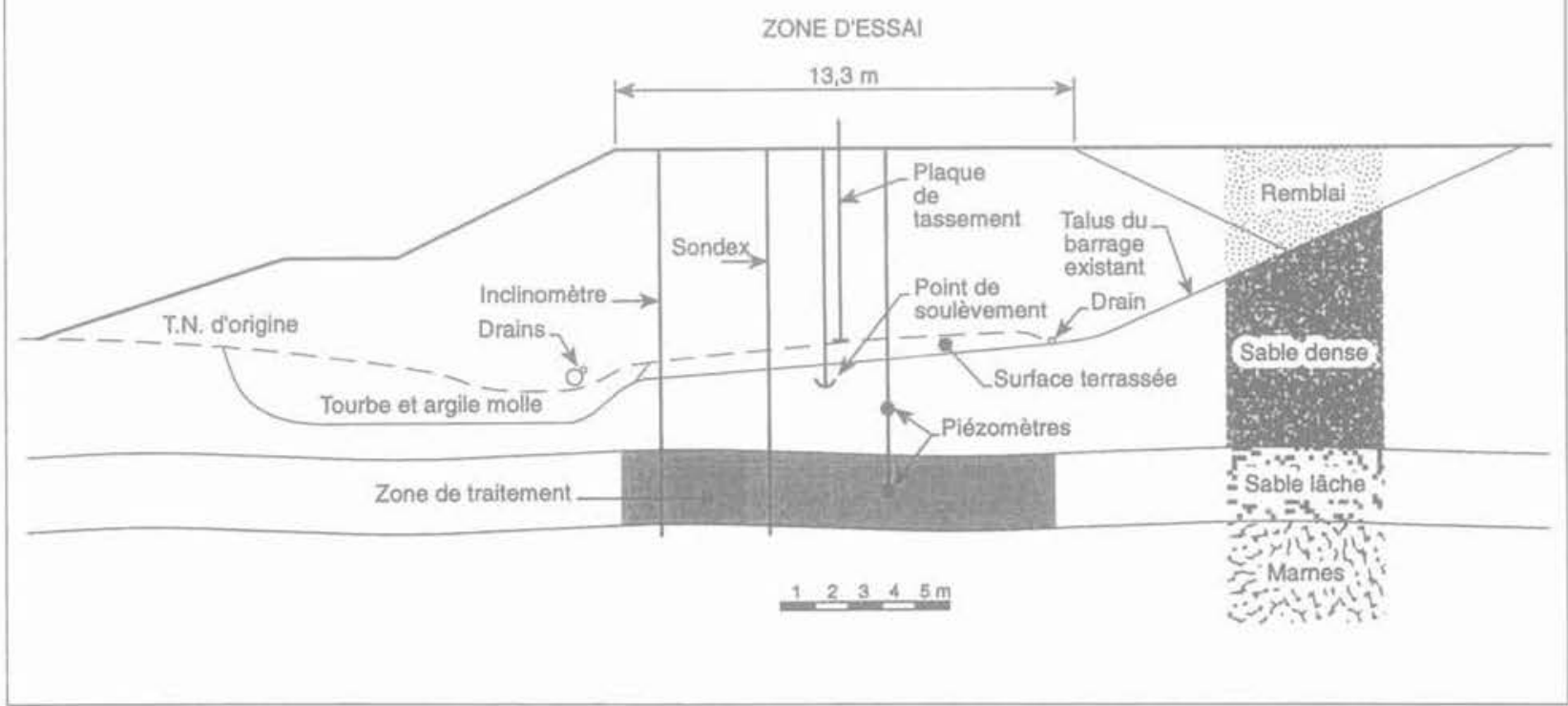

Données issues de Baker. (1985) et Salley et al. (1987) 


\section{APPLICATION 5 : INJECTION DE COMPENSATION}

\section{Chantier}

Seattle Transit Tunnels.

\section{Maître d'ouvrage}

Municipality of Metropolitan Seattle (METRO).

\section{Le projet}

Construction d'un double tunnel pour la circulation des bus en plein centre ville dans le cadre du projet Downtown Seattie Transit Project (DSTP). Diamètre des tunnels $6.5 \mathrm{~m}$, longueur $1525 \mathrm{~m}$, réalisés à environ $12 \mathrm{~m}$ de profondeur.

\section{But de l'intervention}

Limiter les tassements au fur et à mesure de l'avancement du double tunnel.

\section{Terrains}

Glaciaires et interglaciaires du Pléistocéne et de l'Holocène, denses et préconsolidés, des moraines aux sables boulants. Présence d'une nappe sur un tiers du tracé.

\section{Traitement}

- Préventif : réalisé au début à partir de la surface au droit des axes des tunnels avec un espacement de $3 \mathrm{~m}$ (sur une ligne), puis depuis le tunnel même, immédiatement à l'arrière du bouclier. ou à $12-15 \mathrm{~m}$ du front de taille, jusqu'à environ $3 \mathrm{~m}$ au-dessus de la voute et avec un espacement de 1,2 à $3.7 \mathrm{~m}$ sur l'axe de la voute.
- De compensation : dès le dépassement des valeurs de tassement suivantes:

- $3,2 \mathrm{~cm} \mathrm{1,5} \mathrm{m}$ au-dessus des voutes des tunnels,

. $0,5 \mathrm{~cm}$ au droit des bâtiments voisins.

\section{Quantités}

\begin{tabular}{|c|c|c|}
\hline \multirow{2}{*}{ Type de sol } & Incorporation & $\begin{array}{c}\begin{array}{c}\text { Incorporation } \\
\text { moyenne }\end{array} \\
\end{array}$ \\
\hline & \multicolumn{2}{|c|}{ I/ml de tunnel } \\
\hline Argile raide et limons & 2 à 700 & 140 \\
\hline Dépôts glaciomarins & 9 à 7500 & 410 \\
\hline Sables et limons denses & 0,9 à 46300 & 730 \\
\hline Moyenne & & 200 \\
\hline
\end{tabular}

\section{Résultats}

Des tassements en surface limités et inférieurs aux prévisions :

- au droit des axes des tunnels : entre 0 et $6 \mathrm{~cm}$ suivant les terrains rencontrés, avec une moyenne de $2 \mathrm{~cm}$; de 0,1 à $4,3 \mathrm{~cm}$ à l'axe du projet,

- au droit des bátiments : entre $0,3 \mathrm{~cm}$ et $0,8 \mathrm{~cm}$, avec une moyenne de $0,5 \mathrm{~cm}$.

On estime que le traitement par injection solide a permis de réduire de 1,5 a 4 fois les tassements.

\section{Commentaires}

- Le mortier ne contenait pas de ciment, ce qui permettait la réutilisation des forages en cas de besoin.

- Le mortier contenait 2 à $8 \%$ de bentonite.

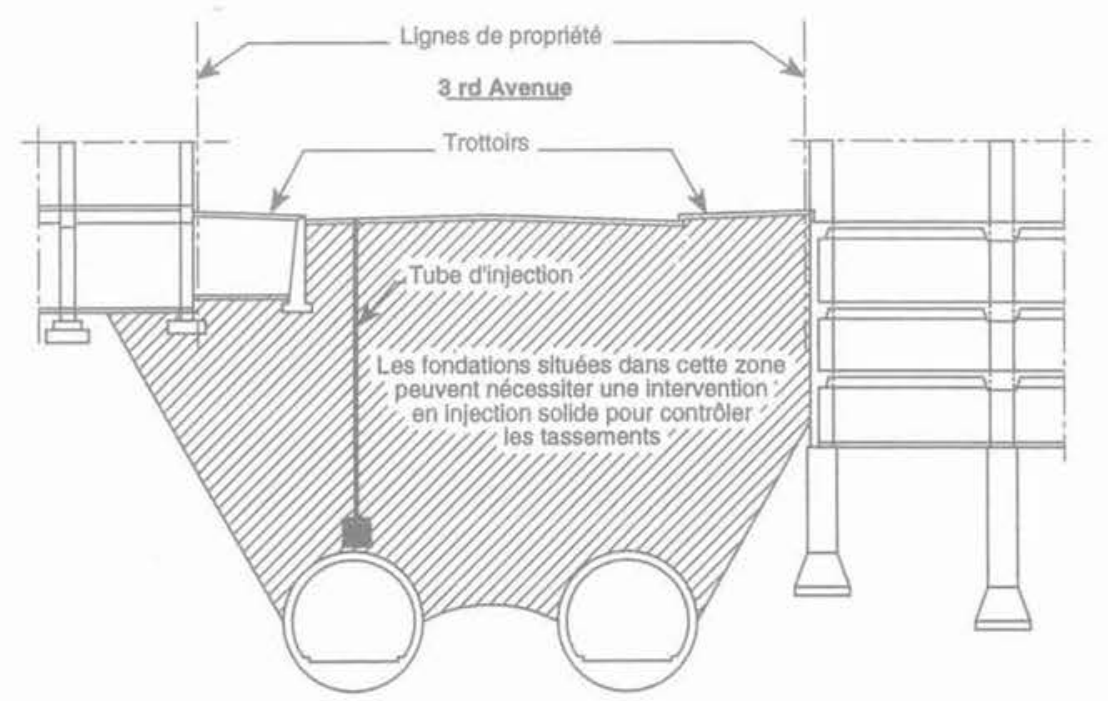

Données issues de Critchfield et al.. (1989) et Robinson et al., (1991) 